\title{
IDENTIFYING GAPS IN AUTOMATING THE ASSESSMENT OF TECHNOLOGY READINESS LEVELS
}

\author{
S. Faidi ${ }^{\otimes}$ and A. Olechowski \\ University of Toronto, Canada \\ $\square$ safa.faidi@mail.utoronto.ca
}

\begin{abstract}
Crucial in the design process, Technology Readiness Levels are a common form of technology maturity assessment. Studies suggest that the TRL scale can be subjective and biased. Automating the assessment can reduce human bias. This paper highlights important challenges of automation by presenting data collected on 15 technologies from the nanotechnology sector. Our findings show that, contrary to claims from the literature, patent data exists for low maturity technologies and may be useful for automation. We also found that there exists unexpected trends in data publications at TRL 2, 3 and 4 .
\end{abstract}

Keywords: technology development, big data analysis, product development

\section{Introduction}

Technology maturity assessment is an important procedure that is crucial for both internal assessment during the product development cycle, and during product planning when assessing potential technologies in which to invest or acquire. One commonly used tool for this assessment is the Technology Readiness Level (TRL) scale developed by NASA in the 1970's (NASA, 2016). The scale consists of nine levels, each level achieved by the technology passing specific testing and demonstration requirements shown in Figure 1.

Technology Readiness Level assessment helps in the design of prototypes. The various stages in the TRL scale create a structure that can better support design development phases (Fast-Berglunda et al., 2014). Through the assessment, professionals can make decisions about the design of the product based on where it stands on the TRL scale shown in Figure 1. Understanding where the technology lies on the scale is important for answering questions like how tests compared with expectations or about the issues encountered during testing which are important inputs in evaluating design (FastBerglunda et al., 2014). Technology assessments are an important part of product development as they provide important checkpoints about where the status of the technology lies. These checkpoints in turn can help designers answer questions needed for prototype development and re-design.

Technology Readiness Level assessment is also used when assessing risk associated with onboarding a technology onto an existing or future product. The various stages in the scale reflect the various degrees of maturity of the technology. Research suggests a significant inverse relationship between Technology Readiness Levels and risks of technology adoption. A technology of lower TRL has a high perceived risk while a technology of higher TRL has a lower perceived risk (Engel et al., 2012). Assessing that risk is important in product planning as it allows product development teams to understand and design the readiness progression scheme. 


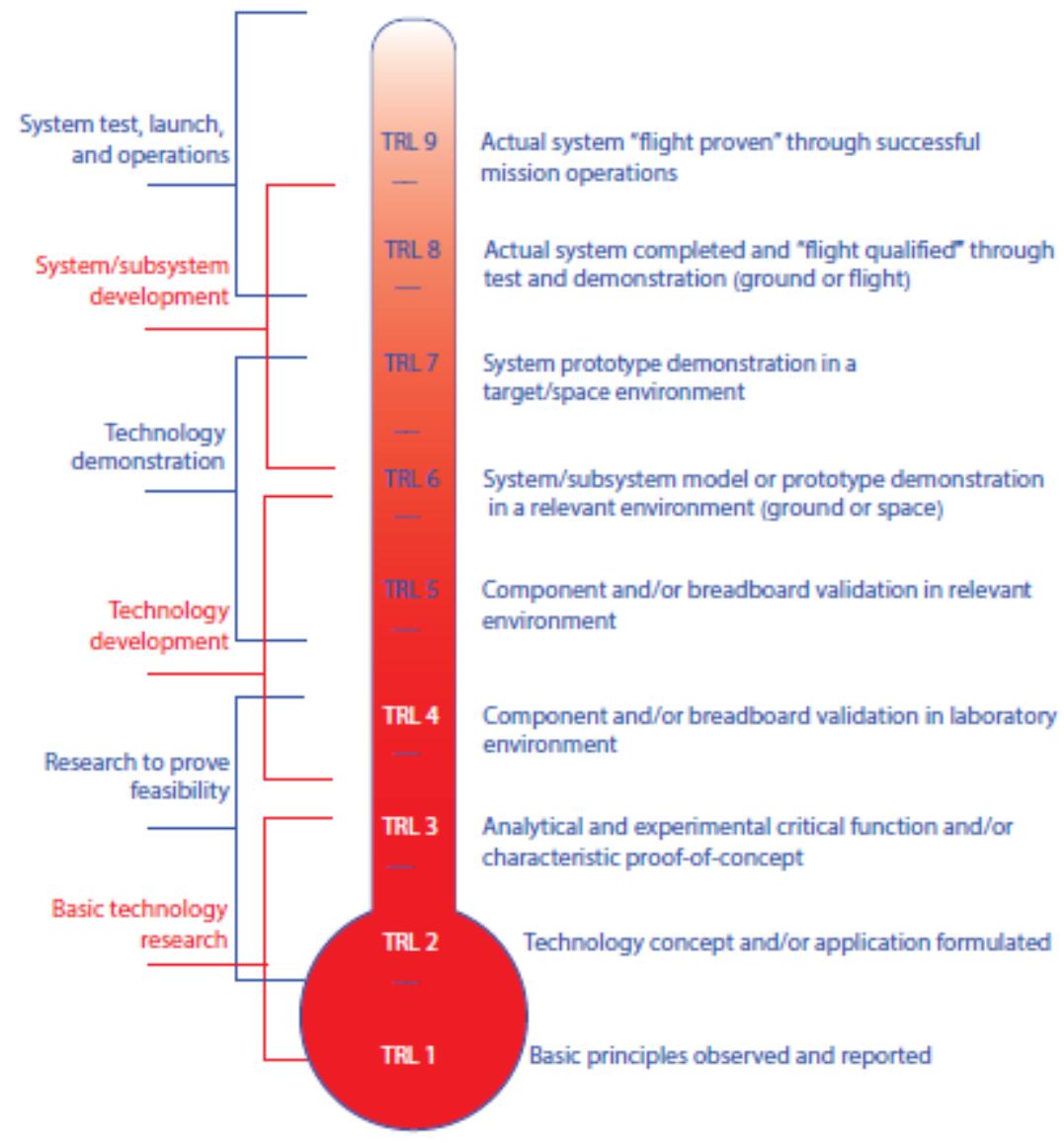

Figure 1. Technology readiness level scale (NASA, 2016)

The Technology Readiness Level scale is widely used. Yet, new evidence suggests that maturity assessment using the TRL scale may suffer from subjectivity and bias (Sarfaraz et al., 2012; LezamaNicolás et al., 2018; Britt et al., 2008). One way to combat this bias is by introducing automation to the assessment. Automation has revolutionized many industries by reducing human input to eliminate subjectivity, bias, and human error. With the current research and work available on machine learning and Artificial Intelligence (AI), many of our existing assessment and design tools could be improved by creating more systematic, reproducible and trust-worthy outputs. Technology assessment is one important area that could be improved if we incorporate the existing machine learning and AI tools into the assessment method. Using big data, we can learn trends and patterns in technology data, and apply them to make judgments about the maturity of a technology.

Our work introduces a small data set to illustrate a number of challenges which will be faced when full automation of the TRL assessment is considered. In particular, we revisit the assertion from the literature that patents and news are not likely explanatory of maturity for emerging (low TRL) technologies. We ask: is the literature correct in pairing specific datasets to specific levels of maturity? Or would a deeper understanding of the patterns of technology documentation across different datasets improve technology maturity assessments?

\section{Background on TRL assessments}

Technology assessments can provide insight about the maturity of a technology which can help organizations make decisions in their $\mathrm{R} \& \mathrm{D}$ planning, product development, or investment decisions (Lokuhitige and Brown, 2017; Watts and Porter, 1997). The Technology Readiness Level scale can either be used to assess a new technology's maturity for deployment in an operational environment or it can be used to assess the risk associated with adding a new technology to an existing system (Engel et al., 2012). The assessment is often carried out by bringing in external specialized consulting teams. Consulting teams would investigate the available reports and data and assess where they believe the 
technology lies on the TRL scale. The decision on where the technology lies on the TRL scale is then finalized by the different groups within the same organization: $\mathrm{R} \& \mathrm{D}$, manufacturing, upper management, etc. This method is often expensive as consulting teams charge companies for their time and assessment.

Previous studies highlighted the issues associated with using the TRL scale (Olechowski, 2017; Sarfaraz et al., 2012; Lezama-Nicolás et al., 2018; Britt et al., 2008). One of the reported issues is the subjectivity of the scale. Many industry professionals reported that the TRL scale assessment can be biased based on each stakeholder's interest (Olechowski, 2017). For example, R\&D may want to spend more time perfecting the technology that they advocate for a lower TRL. In addition, the different levels in the scale may be interpreted differently amongst a team. Both issues are due to the low level of detail provided by the scale for each level. These issues push decision-making to be more time consuming and based on inaccurate input.

Automating the TRL scale can provide a non-biased, subjective, and repeatable method to assess technologies. Automation could also drastically decrease the time it would take to assess a technology. This will in turn provide great financial benefit to corporations assessing technologies and can transform the technology assessment industry. Automating technology assessments can take place by analysing data from datasets like patents, scientific literature, and news, and matching the trend in data to the corresponding TRL.

There are however complications and challenges in automating an assessment method like the TRL scale. Since the scale is made to be used for all industries, the varying industries may need to be taken into consideration. Each industry could have a differing effect on how data appears in different datasets. For example, can we assume that a technology that adopts AI will have the same pattern of data in the news as a technology that uses a more traditional technology? Or do industries need to be taken into consideration when we assess which datasets are more likely to reveal how mature a technology really is?

Another challenge in automating the assessment method is finding the appropriate type of data to use for identifying common patterns. Technology behaviour can often be studied in very different datasets. These datasets include patent documents, scientific publications, industry news, clinical trials, research projects and more. Machine learning requires training models using large data sets. The challenge lies in choosing the correct dataset that will have the strongest correlation with a technology's maturity.

\section{Previous work on automating TRL assessments}

There have been previous efforts to automate the Technology Readiness Level assessment. LezamaNicolás et al. use a bibliometrics method to assess technological maturity (Lezama-Nicolás et al., 2018) Bibliometrics is a well-known method often used for technology forecasting. The method uses counts of publications from different datasets like scientific papers or patents to interpret the maturity of the technology under assessment. In their paper, Lezama-Nicolás et al. use the varying stages in the Technology Life Cycle Curve: emerging, growing, and mature in their assessment. The assessment will use the amount of data available for that technology in order to estimate the Technology Readiness Level. If the technology lies in the emerging phase, the bibliometrics assessment method will use scientific papers and engineering papers as their main data source. If the technology lies in the growing phase, the main dataset source is patents. If the technology lies in the mature phase, then the main dataset source is news records. The authors highlight that if the technology is emerging then the assigned TRL will be between 1-5, if it's growing then the assigned TRL will be either 6 or 7, and if it's mature then the assigned TRL will be either 8 or 9 .

Much of the other previous work highlighted that emerging technologies, or technologies with lower maturities, show more activity in scientific literature than patents and news (Lokuhitige and Brown, 2017; Watts and Porter, 1997; Lezama-Nicolás et al., 2018). Understanding which datasets give insightful input about the maturity status of a technology is crucial before attempting to automate it. For this reason, this paper challenges the prior assumptions from the literature regarding the match of the dataset to the maturity of the technology under assessment. We present data on 15 technologies of low TRL to investigate how technologies of lower maturity behave in datasets other than the scientific literature dataset. We investigate their behaviour in patents and news, which are datasets often used only when studying technologies of higher maturity (high TRL). 


\section{Methods}

\subsection{Technologies}

We focused the analysis on 15 technologies in the nanotechnology sector. The technologies were chosen from the 2015 NASA technology roadmaps (NASA, 2015). The NASA roadmaps provide an overview of selected technologies that NASA views as candidates that could support future missions, but also may support applications for the general population. The roadmaps examine development pathways of these technologies for a span of 20 years (2015-2035). One section of the roadmaps lists the achieved Technology Readiness Level of each technology. In 2015, the roadmaps explicitly included an assessed TRL, thus our assessment was carried out using the technologies listed in NASA's 2015 technology roadmaps for its nanotechnology sector.

There are 77 technologies included in the nanotechnology roadmap. For the explanatory purposes of our pilot, we chose to focus on 15 nanotechnologies: five technologies of TRL 2, five technologies of TRL 3 and five technologies of TRL 4 (see Table 1). We excluded TRL 1 from our search because we believe the maturity may be too low for publication activity. We also excluded TRL 5 from the assessment because the NASA nanotechnology roadmaps only listed one technology with TRL 5 .

Table 1. Technologies selected for study in this work

\begin{tabular}{|c|c|c|}
\hline TRL 2 & TRL 3 & TRL 4 \\
\hline Nanostructured Thermal Sink & $\begin{array}{c}\text { Nanotube Reinforced Structural } \\
\text { Composite }\end{array}$ & Low Permeability Nanocomposites \\
\hline Low Density Nanofiber & Graphene Sheets & Nanocomposite Ablators \\
\hline Damage Sensing Nanocomposite & Nanostructured Coatings & $\begin{array}{c}\text { Lithium (Li) Battery Solid Polymer } \\
\text { Electrolytes }\end{array}$ \\
\hline Nanotube Composite Flywheel & Biomimetic Adhesive & Aerogel Cryotank Insulation \\
\hline Nanostructured Supercapacitor & Nanolithography & Fouling Resistant Surfaces \\
\hline
\end{tabular}

\subsection{Data access}

We next sought a preliminary understanding of the data sets of the selected technologies. The data was collected using the Application Program Interface (API) for the commercial software Mergeflow. Mergeflow's database is made up of 10 datasets: scientific publications, patents, industry news, investor news, technology blogs, venture capital, technology licensing, funded research projects, startups, and clinical trials, each consisting of thousands of documents updated weekly. Mergeflow's scientific publications dataset consists of research papers, conference proceedings and preprints from different disciplines. It includes databases such as arxiv and PubMed. The patent dataset consists of patent publications from worldwide patent offices. In addition, the industry news dataset consists of worldwide business news from different industries. Our data was collected for 7 years prior to 2015 . For the purpose of this paper, we chose to analyze data from three datasets: patents, scientific publications, and industry news. This is because previous literature only used patents, scientific publications and industry news for their analysis, and the purpose of this study is to compare our findings to that of previous literature.

For each technology, we formed a search query including the following elements:

- We used the technology title provided by NASA as the basis of the search. We modified the title with Mergeflow's wildcard searches when it was necessary to broaden the word endings.

- We also included the "technology areas" listed by NASA in the roadmaps, for example: avionics, autonomy, information technology, radiation, and space. Each industry was separated by an "OR" statement and had wildcards incorporated in their query to include all possible variations of the word. 


\section{Results and discussion}

Table 2 and Table 3 show the average document count for TRL 2 and TRL 4 technologies, respectively. Each table contains the average document count for all five technologies at each year. Due to their low document count, these technologies were not plotted.

Table 2. Average behaviour in datasets of TRL 2 technologies listed in Table 1

\begin{tabular}{|l|l|l|l|l|l|l|l|l|}
\hline & \multicolumn{5}{|c|}{ Average Document Count } & \\
\hline & 2015 & 2014 & 2013 & 2012 & 2011 & 2010 & 2009 & $\begin{array}{l}\text { Average document count in all } \\
\text { reported years }\end{array}$ \\
\hline Patent Documents & 0 & 0.2 & 0 & 0 & 0.6 & 0.2 & 0 & 0.14 \\
\hline $\begin{array}{l}\text { Scientific } \\
\text { Publications }\end{array}$ & 2 & 3.2 & 0 & 0.4 & 0 & 0.4 & 0 & 0.86 \\
\hline Industry News & 1.2 & 0.2 & 0.4 & 0 & 0 & 0.2 & 0 & 0.29 \\
\hline
\end{tabular}

Table 3. Average behaviour in datasets of TRL 4 technologies listed in Table 1

\begin{tabular}{|l|l|l|l|l|l|l|l|l|}
\hline & \multicolumn{7}{|c|}{ Average Document Count } & \\
\hline & 2015 & 2014 & 2013 & 2012 & 2011 & 2010 & 2009 & $\begin{array}{l}\text { Average document count in all } \\
\text { reported years }\end{array}$ \\
\hline Patent Documents & 0.4 & 0.6 & 0 & 0 & 0.2 & 0.6 & 0 & 0.26 \\
\hline $\begin{array}{l}\text { Scientific } \\
\text { Publications }\end{array}$ & 0.4 & 0.8 & 0.2 & 0 & 0 & 0 & 0 & 0.23 \\
\hline Industry News & 0.6 & 0 & 0 & 0 & 0 & 0.2 & 0 & 0.11 \\
\hline
\end{tabular}

TRL 2 technologies and TRL 4 technologies showed low activity in all three datasets (see Table 2 and Table 3). The document counts for TRL 2 and TRL 4 technologies were much lower than for TRL 3 technologies. TRL 2 technologies are defined as technologies that only have their concept and/or application formulated. This means that the technologies are yet to have their concepts tested or proven. This may explain why they scored low in patent and scientific publication documents with negligible document counts respectively over a span of 7 years. TRL 2 technologies also scored low in industry news. This may be explained due to the low popularity of a technology that only has a concept formulated with no practical application identified.

TRL 4 technologies are defined as technologies that have had their prototypes validated in laboratory environment. We expected TRL 4 technologies to show more activity (higher document count) in the datasets than TRL 2 and TRL 3 technologies, however that was not what our results show. TRL 4 technologies showed lower activity in scientific publications and industry news compared to TRL 2 technologies. A potential explanation for this may be related to the specificity of the technology name and corresponding search query. We hypothesize that technologies become more specific in their descriptions and titles as they progress through the TRLs. When technologies are only concepts, they might have more general names due to the low level of detail associated with low maturity technologies. However, as technologies progress through the TRLs, their application and function become more specific, and more details might be added to their description which can ultimately change the way the technology is identified. Since we used the NASA roadmaps' technology names explicitly in our search query, we may see lower document counts for TRL 4 when compared to TRL 3 because the TRL 4 
technologies have a more specific name, thus resulting in a narrower search query. One example of this can be seen in Table 1. Technologies listed under TRL 3 have more general names than technologies listed under TRL 4. For example, "Aerogel Cryotank Insulation" is more specific in its description of the technology where both the material and the function are identified in its name. On the other hand, technologies like "Graphene Sheets" have a more general description. This hypothesis needs further research and presents itself as an interesting question for future work.

Figures 2-4 represent data collected on the five nanotechnologies listed in Table 1 of TRL 3 maturity in 2015. Figure 2 shows the behaviour of five nanotechnologies of TRL 3 over the span of 7 years in the patent dataset. All technologies, except for Nanolithography, showed an increase in patent documents from 2009 to 2015. Contrary to the conclusions from existing literature, our results show that patent data may provide useful insight when assessing the maturity of low TRL (emerging) technologies. TRL 3 technologies are described as technologies that have had a proof-of-concept through laboratory experiments or analytical studies. This could be a significant time for these concepts to be patented which could explain the rise in patents in 2015, the year of the TRL 3 assessment.

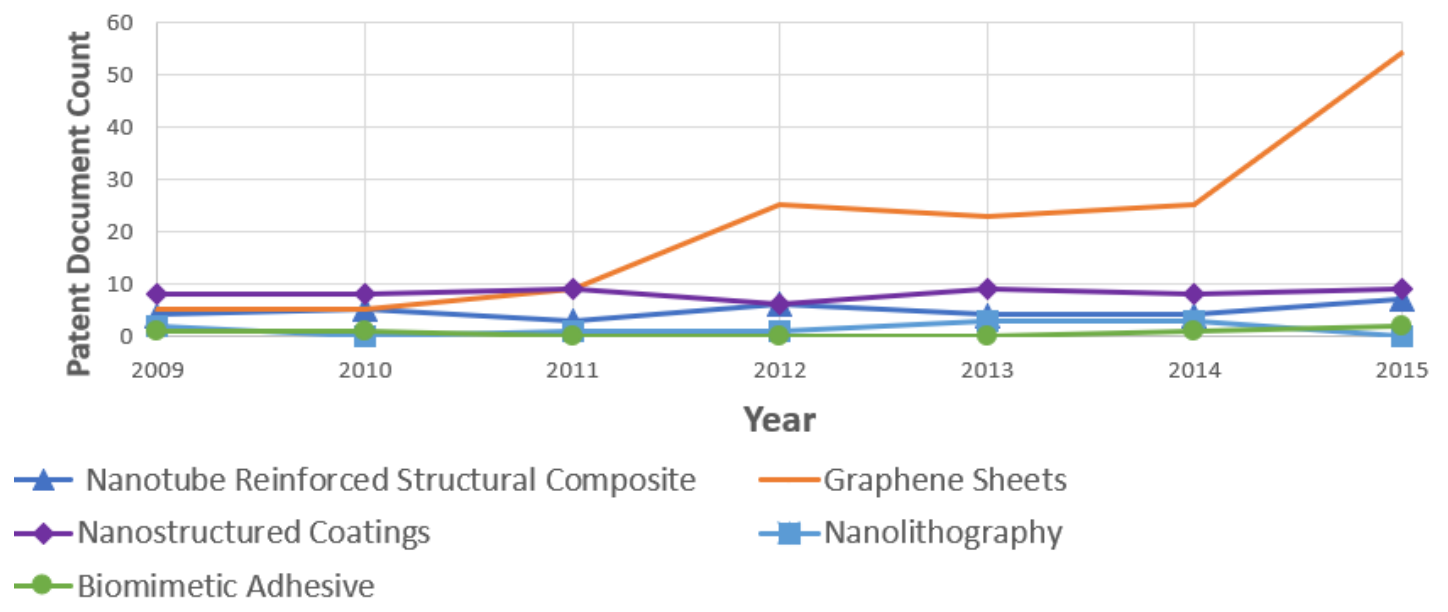

Figure 2. Patent counts for TRL 3 technologies

Figure 3 represents the pattern for five technologies of TRL 3 over the span of 7 years in scientific publications. All five technologies showed an increase in publications from 2009 to 2015. This trend agrees with previous literature work (Lokuhitige and Brown, 2017; Watts and Porter, 1997; LezamaNicolás et al., 2018).

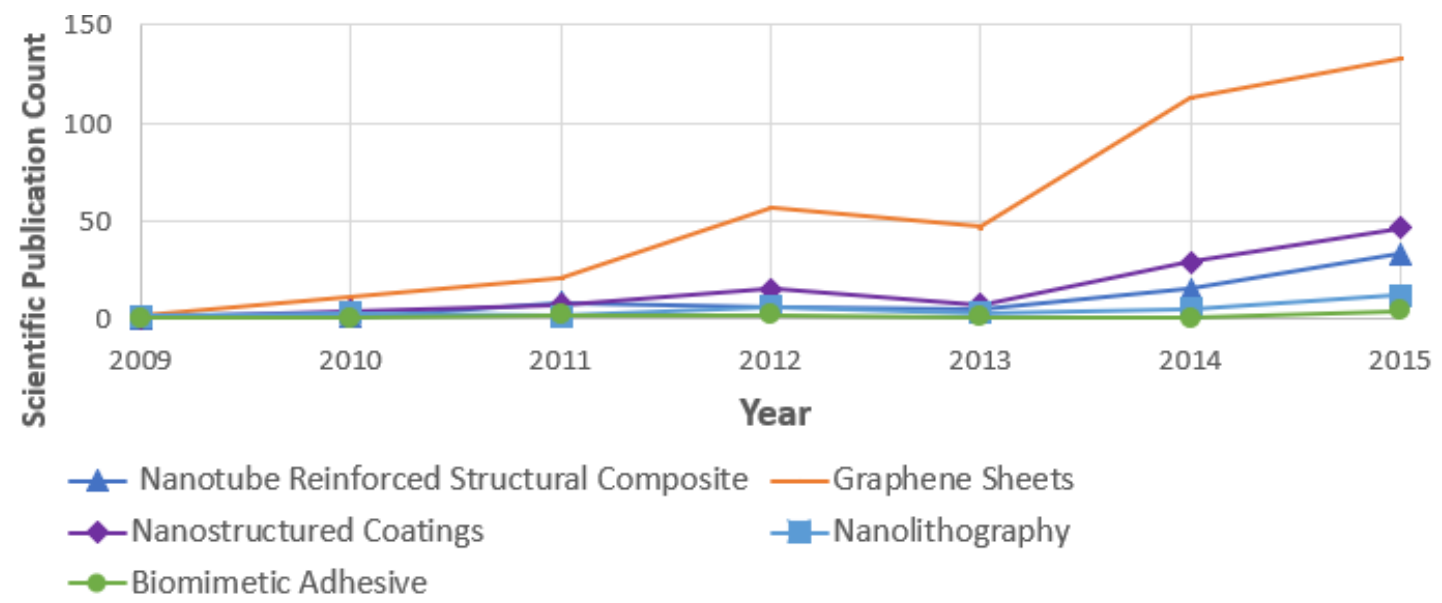

Figure 3. Scientific publication counts for TRL 3 technologies

Figure 4 shows the pattern for the same five TRL 3 technologies in industry news. Three of the five technologies showed significant increase in the number of news stories from 2009 to 2015. These 
findings do not match the anticipated trends described in the literature. News trends in the literature are often described as an important signal only for mature technologies (TRL 8-9) (Lezama-Nicolás et al., 2018). However, our results reveal activity existing at emerging technologies of lower maturity which could be useful to consider when drawing conclusions prior to automating the TRL scale.

One interesting finding to highlight is that four of the five technologies showed similar trends in the increase of document count from 2009 to 2015 in patents and scientific literature. In both datasets, Graphene sheets had the highest publications and patent documents than any other technology. This was followed by Nanostructured coatings which also had a similar increase between publications and patent documents. This existing link between patent behaviour and scientific publications behaviour can be useful input when attempting to automate the TRL scale.
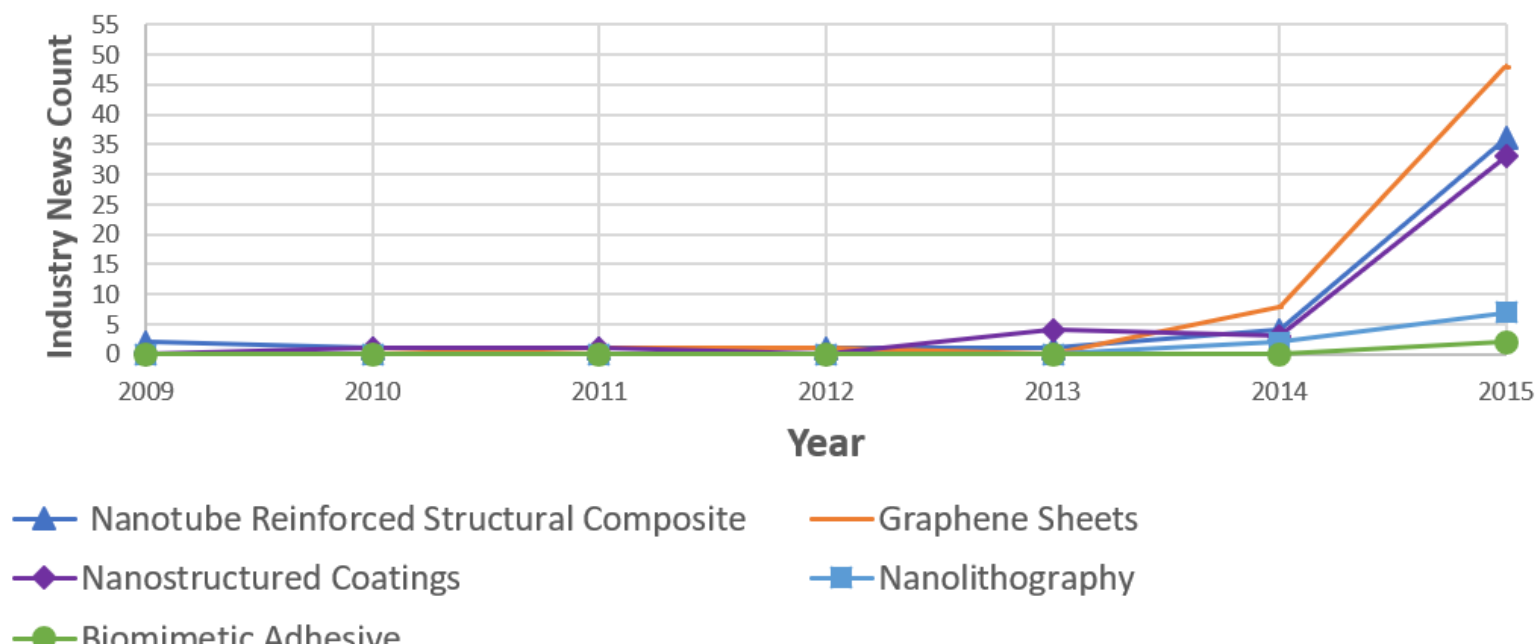

Figure 4. Industry news counts for TRL 3 technologies

This study focused on understanding the document count trends of low maturity TRLs; We studied possible patterns for the TRLs that represent low maturity and not a comparison between emerging technologies and mature technologies and will extend to higher maturity levels in future research. We can then make comparisons between levels and highlight additional information that can be useful to take into consideration when attempting to automate technology assessments.

\section{Conclusion}

Technology assessments are an important step in product development and design. Understanding where a technology stands in its maturity can help designers make decisions when evaluating their designs. The Technology Readiness Level is a commonly used maturity assessment tool but was reported to be subjective and biased. Previous work tried to solve this subjectivity by transforming the assessment to be more automated. However, some gaps still exist in what input we should use when assessing maturity through automation rather than human input. This paper presented data from 15 technologies in the nanotechnology sector that were assessed in 2015 at TRL2, TRL 3 and TRL 4. We analysed the data to study the technologies' behaviour in three datasets: patents, scientific publications, and industry news.

Our findings prove that patent data behaviour is significant in TRL 3 technologies and may represent an important additional data input for classification. We also found that TRL 3 patent behaviour trends are similar to TRL 3 scientific publications trend. This could be used when building a classification model that uses data patterns to predict where a technology stands on the Technology Readiness Level scale. Our findings also show unexpected trends for TRL 4 technologies. This could be explained by the possibility of technologies having more specific names when they progress through maturity. However, we believe this requires further research. Our findings can provide additional input that can be useful for making the automated tool universal by considering possible changes in technology specifications as they mature.

There still is a gap in understanding whether industry influences the behaviour of technologies in different datasets. Do medical devices, for example, show similar patterns in patents as nanotechnologies? Or is 
industry a factor that must be taken into consideration when attempting to build a universal automated tool? This is one of the motivations for our future research. We believe that a comparison between the behaviour of technologies from different sectors will provide crucial input when building a classification model. This comparison may require normalisation of our data to account for the difference in the field sizes of different technologies. In addition, future work will involve statistical tests to formally identify differences in trends.

\section{Acknowledgments}

The data collected in this work was provided through granted access to Mergeflow's API. We would like to thank Dr. Florian Wolf for this access and for his support.

\section{References}

Britt, B.L. et al. (2008), "Document Classification Techniques for Automated Technology Readiness Level Analysis", Journal of the American Society for Information Science and Technology, Vol. 59 No. 4, pp. 675-680. https://doi.org/10.1002/asi

Engel, D.W. et al. (2012), "Development of Technology Readiness Level (TRL) Metrics and Risk Measures".

Fast-Berglunda, A. et al. (2014), "Using the TRL-methodology to design supporting ICT-tools for production operators", Elsevier, pp. 726-731. https://doi.org/10.1016/j.procir.2014.02.039

Lokuhitige, S. and Brown, S. (2017), "Forecasting Maturity of IoT Technologies in Top 5 Countries Using Bibliometrics and Patent Analysis", In Proceedings - 2017 International Conference on Cyber-Enabled Distributed Computing and Knowledge Discovery, CyberC 2017, pp. 338-341. https://doi.org/10.1109/ CyberC.2017.35

Lezama-Nicolás, R. et al. (2018), “A bibliometric method for assessing technological maturity: the case of additive manufacturing”, Scientometrics. Springer International Publishing, Vol. 117 No. 3, pp. 1425-1452. https://doi.org/10.1007/s11192-018-2941-1

NASA (2016), NASA System Engineering Handbook SP-2016-6105 Rev2. Rev2 edn. Edited by D. Hoffpauir. Available at: http://hdl.handle.net/2060/20170001761

NASA Technology Roadmaps TA 10: Nanotechnology (2015), Available at: https://www.nasa.gov/sites/default/ files/atoms/files/2015_nasa_technology_roadmaps_ta_10_nanotechnology_final.pdf

Olechowski, A. (2017), Essays on decision-making in complex engineering systems development. https://doi.org/10.1007/s11661-014-2485-9

Sarfaraz, M., Sauser, B.J. and Bauer, E.W. (2012), "Using System Architecture Maturity Artifacts to Improve Technology Maturity Assessment”, Procedia Computer Science, pp. 165-170. https://doi.org/10.1016/ j.procs.2012.01.034

Watts, R.J. and Porter, A.L. (1997), "Innovation Forecasting”, Technological Forecasting and Social Change, Vol. 56 No. 1, pp. 25-47. https://doi.org/10.1016/S0040-1625(97)00050-4 